\title{
Choroidal Detachment Following Multi-spot Double Frequency Nd-YAG Retinal Photocoagulation - A Case Report
}

\author{
Sangeetha Rajagopal ${ }^{*}$, Nivean Madhivanan, Lakshmi Mayilvakanam, Pratheebadevi Nivean
}

MN Eye Hospitals Pvt Ltd, Chennai, India

\section{*Correspondence to}

Sangeetha Rajagopal,

Consultant-Medical Retina, MN Eye Hospitals Pvt Ltd, Chennai, India.

Tel: +919176288898;

Email: sangeetharajagopal03@ gmail.com

Published online June 21, 2020

\begin{abstract}
Introduction: Panretinal photocoagulation (PRP) remains the gold standard treatment for severe non-proliferative and proliferative diabetic retinopathy (PDR), as it reduces the risk of severe visual loss by more than $50 \%$. In the conventional single-spot laser, the procedure involves the application of moderate-intensity burns of 200-500 microns, placed one spot-size apart to achieve a total of 1200-2000 applications in 2 or 3 sessions. The more advanced retina lasers like the Pattern Scan Laser (PASCAL) and the VITRA multi-spot laser are $532 \mathrm{~nm}$ frequency-doubled (Nd: YAG) solid-state lasers. These modern lasers enable the application of multiple laser burns in a rapid pre-determined sequence with reduced pulse duration $(10-20 \mathrm{~ms})$ to facilitate the PRP to be completed in a single sitting with lesser collateral tissue damage.

Case Report: Though multi-spot lasers have significantly reduced the adverse events when compared with the conventional single-spot lasers, we report a case of rare adverse events (serous choroidal detachment) following PRP with the VITRA multi-spot double frequency NdYAG (532 nm) laser.

Conclusion: Most of the serious choroidal detachments following PRP are self-limiting. We recommend complete retinal evaluation post laser procedure even with modern multisport laser to look for such adverse events.

Keywords: Panretinal photocoagulation; Multi-spot lasers; Choroidal detachment.
\end{abstract}

\section{Introduction}

With diabetes now regarded as an epidemic, diabetic retinopathy remains the leading cause of blindness worldwide. ${ }^{1}$ The treatment of vision-threatening diabetic retinopathy involves retinal laser photocoagulation ${ }^{2}$ and intravitreal anti-VEGF/steroid injections. The invention of intravitreal anti-vascular endothelial growth factors has revolutionized the treatment of diabetic retinopathy, but retinal photocoagulation still remains the gold standard treatment for diabetic retinopathy. With the advent of newer laser technologies and treatment techniques, retinal photocoagulation outweighs other treatment modalities in the treatment of diabetic retinopathy.

Though uncommon, we report a case of localised serous choroidal detachment following VITRA multispot double frequency Nd-YAG photocoagulation in a patient with proliferative diabetic retinopathy, which was self-limiting. As most of the serous choroidal detachments after panretinal photocoagulation (PRP) are subclinical and self-limiting, the reported incidence of such adverse events could be less.

\section{Case Report}

A 55-year-old male with type 2 diabetes presented with a history of defective vision in both eyes. Best-corrected visual acuity in both eyes was 20/40. Ocular examination revealed a peripheral cortical cataract and proliferative diabetic retinopathy (PDR) with clinically significant macular edema in both eyes (Figure 1). The spectral domain optical coherence tomography (SD-OCT showed cystoid macular edema in both eyes. The central subfield thickness was 332 microns in the right eye and 392 microns in the left eye. The fasting blood glucose level was $248 \mathrm{mg} / \mathrm{dL}$ and the post-prandial blood glucose level was $350 \mathrm{mg} / \mathrm{dL}$, with the HbA1c level being 8.5\%. The patient underwent pan-retinal photocoagulation with the VITRA multi-spot laser delivery system. Intravitreal anti-VEGF injection for centre-involving diabetic macular edema was planned after the control of blood sugar.

Pan-retinal photocoagulation in the right eye was carried out with the VITRA multi-spot laser (double frequency Nd-YAG). The laser parameters for the PRP included a spot size of 200 microns, at $225 \mathrm{~mW}$ power 

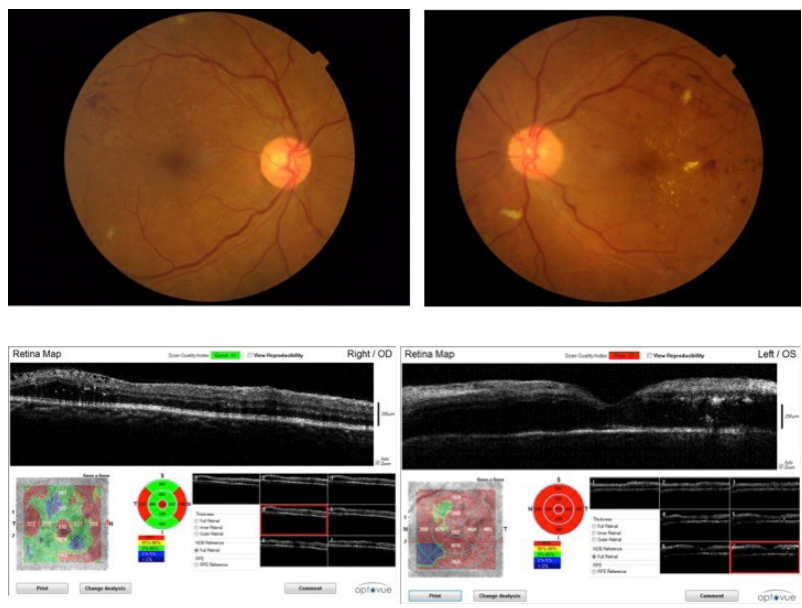

Figure 1. Fundus Picture of Right and Left Eyes With SD-OCT Macula Showing Cystoid Macular Edema.

for the duration of $25 \mathrm{~ms}$, creating a total of 1250 burns covering the superior quadrant.

Five days after the procedure, the best corrected visual acuity worsened to 20/80 in the right eye. He developed peripheral localised choroidal detachment in the superior quadrant and worsening of diabetic macular edema (Figure 2). The ultrasound B-scan of the right eye revealed smooth dome-shaped localised choroidal detachment with anechoic supra-choroidal space. The corresponding A-scan showed M-shaped double peak reflectivity, suggestive of localised serous choroidal detachment in the superior quadrant of the right. The central subfield thickness by SD-OCT was 404 microns in the right eye. He was prescribed topical prednisolone acetate 1\% QID in the right eye for one week, with good glycemic control.

When reviewed after one week, the right eye showed a complete resolution of the choroidal detachment (Figure 3). The ultrasound B-scan of the right eye confirmed the complete resolution of the choroidal detachment. However, the cystoid macular edema and the visual acuity remained unchanged from the last review.

\section{Discussion}

Diabetic retinopathy remains the leading cause of blindness worldwide. ${ }^{1}$ The new vessels arising on the optic disc and elsewhere in PDR tend to bleed due to the contraction of the overlying vitreous gel.

If not treated, they progress to advanced pathologies like tractional retinal detachment, combined tractional/ rhegmatogenous retinal detachment and rubeosis iridis. This causes a reduction in central and peripheral vision. The Early Treatment Diabetic Retinopathy Research Group (ETDRS) recommends that scatter PRP should be initiated in severe retinopathy and should not be delayed if the eye has already reached the highrisk proliferative stage. ${ }^{2}$ It also suggests that focal laser photocoagulation is effective in reducing the risk of moderate visual loss in persistent macular edema. ${ }^{3}$ The
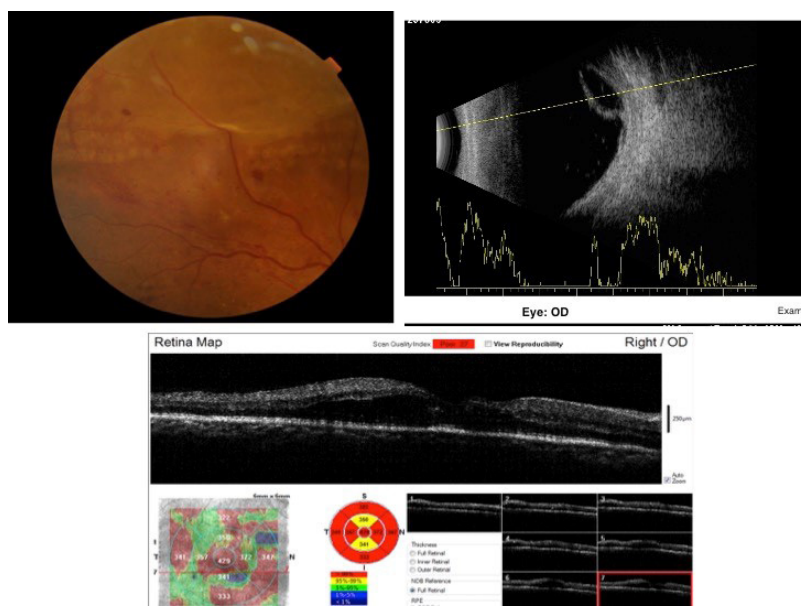

Figure 2. Fundus Picture of the Right Eye Showing Localized Choroidal Detachment in the Superior Quadrant With the Corresponding B Scan. SD-OCT showing worsening of macular edema.

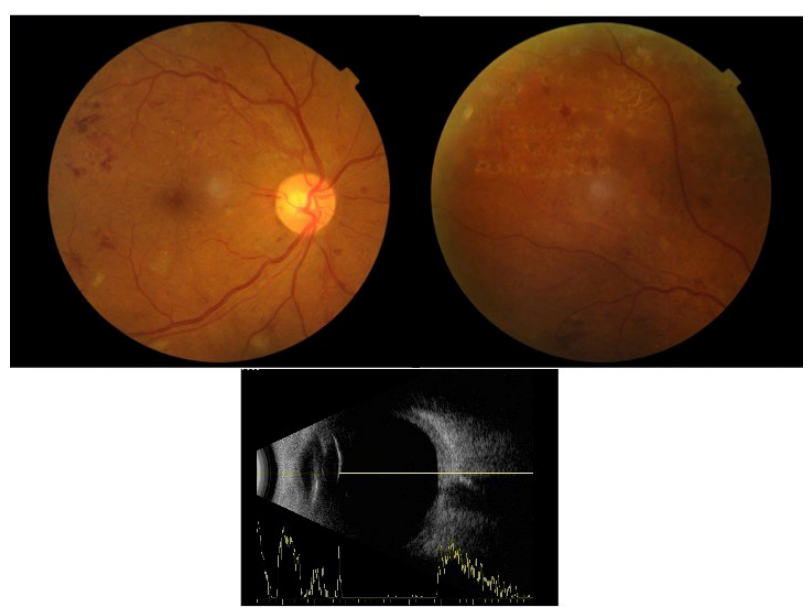

Figure 3. Fundus Picture and B Scan Showing Complete Resolution of the Choroidal Detachment.

Diabetic Retinopathy Study Research Group ${ }^{4}$ has clearly proved that photocoagulation treatment is of benefit in preventing severe visual loss in PDR over a period of twoyear follow-up.

The aim of pan-retinal photocoagulation treatment is to preserve central vision and prevent further loss in vision due to progressive diabetic retinopathy. Laser application creates thermal burns in the peripheral retina, leading to tissue coagulation. This improves the anti-VEGF levels and retinal oxygenation. There are certain adverse events associated with conventional PRP therapy, such as visual field defects, difficult night vision, epiretinal membrane formation. Increased dispersion of thermal energy within the retina and choroid can cause increased macular edema, choroidal effusion and exudative retinal detachment. ${ }^{5}$ The risk of these complications is closely associated with the laser parameters like increased power and duration and the number of burns applied in a single 
sitting.

But with the advent of multi-spot lasers, the laser photocoagulation is made safer, more effective, easier and more convenient for the patient and the physician. The Vitra multi-spot (Quantel medical, France) laser is a $532 \mathrm{~nm}$ photocoagulator that is characterized by the use of short pulse duration (10-20 ms). ${ }^{6}$ It offers many advantages over classical single-spot treatments by less heat diffusion to the choroid and retina, reduced collateral tissue damage, lessened inflammatory response and lesser damage to the retinal nerve fiber layer. ${ }^{7}$ Clinically, patients' comfort is more than the conventional laser due to the decreased energy delivered to the retina with limited thermal spread to the choroid.

Although the short pulse duration of the multi-spot laser requires the use of high power, not many adverse effects have been reported. ${ }^{8}$ However, we report a case of choroidal detachment following the multi-spot laser. This could be caused by the increased inflammation following PRP, causes alterations in the blood retinal barrier and capillary basement membrane. ${ }^{9}$ Though most of the serous choroidal or retinal detachment following PRP are self-limiting, one has to be aware of this potential complication, especially in patients with poorly controlled diabetes or with nephropathy.

\section{Conclusion}

The multi-spot lasers are considered superior to the conventional lasers due to its increased safety, patient comfort, precision of laser delivery and control. However, the risk of choroidal detachment and worsening of macular edema with multi-spot lasers still exists. The aim of this case report is to make the physician and the patient aware of this potential, though uncommon adverse events could happen even with modern multispot lasers. The ideal laser parameters and the maximum tolerated spot count per session in multi-spot lasers are not yet determined. Proper counseling of the patient on this potential complication before the initiation of the treatment is essential. Although most of the serous choroidal detachments following PRP are self-limiting, complete retinal evaluation after the procedure to look for such adverse events should be done.

\section{Ethical Considerations}

Written informed consent was obtained from the patient for publishing this case report.

\section{Conflict of Interests}

The authors have no conflict of interest to declare.

\section{References}

1. Ding J, Wong TY. Current epidemiology of diabetic retinopathy and diabetic macular edema. Curr Diab Rep. 2012;12(4):346-54. doi: 10.1007/s11892-012-0283-6.

2. Early Treatment Diabetic Retinopathy Study Research Group. Early photocoagulation for diabetic retinopathy: ETDRS report number 9. Ophthalmology. 1991;98(5):76685. doi: 10.1016/S0161-6420(13)38011-7.

3. Early Treatment Diabetic Retinopathy Study Research Group. Photocoagulation for diabetic macular edema: Early Treatment Diabetic Retinopathy Study Report Number 1. Arch Ophthalmol. 1985;103(12):1796-806. doi:10.1001/ archopht.1985.01050120030015.

4. The Diabetic Retinopathy Study Research Group. Preliminary report on effects of photocoagulation therapy. Am J Ophthalmol. 1976;81(4):383-96. doi: 10.1016/00029394(76)90292-0.

5. Deschler EK, Sun JK, Silva PS. Side-effects and complications of laser treatment in diabetic retinal disease. Semin Ophthalmol. 2014;28(5-6):290-300. doi: 10.3109/08820538.2014.959198.

6. Nagpal M, Marlecha S, Nagpal K. Comparison of laser photocoagulation for diabetic retinopathy using 532-nm standard laser versus multispot pattern scan laser. Retina. 2010;30(3):452-8. doi: 10.1097/IAE.0b013e3181c70127.

7. Muqit MM, Marcellino GR, Henson DB, Young LB, Patton $\mathrm{N}$, Charles SJ, et al. Single-session vs multiple-session pattern scanning laser panretinal photocoagulation in proliferative diabetic retinopathy: The Manchester Pascal Study. Arch Ophthalmol. 2010;128(5):525-33. doi: 10.1001/ archophthalmol.2010.60.

8. Salman AG. Pascal laser versus conventional laser for treatment of diabetic retinopathy. Saudi J Ophthalmol. 2011;25(2):175-179. doi: 10.1016/j.sjopt.2011.01.006.

9. Natesh S, Ranganath A, Harsha K, Yadav NK, Bhujang BS. Choroidal detachment after PASCAL photocoagulation. Can J Ophthalmol. 2011;46(1):91. doi: 10.3129/i10-108. 